\title{
Q\&A
}

\section{iPOP and its role in participatory medicine}

\author{
Michael Snyder
}

\section{Abstract \\ Michael Snyder shares his thoughts on participatory medicine and how omics profiling could fit into this new model of healthcare where patients are at the center of medicine.}

\section{Introduction}

Michael Snyder (Figure 1) is the Chair of the Genetics Department at Stanford University, California, USA, and the Director of the Stanford Center for Genomics and Personalized Medicine. Last year, his team published a landmark article in Cell that presented Snyder's own integrative personal omics profile (iPOP) [1]. His experience as a participant in genomic medicine and as a researcher put him in a unique position to discuss participatory medicine.

\section{Last year you published a study about personal omics} profiling. Could you briefly describe what this is?

Personal omics profiling involves trying to bring together as many measurements as possible with the goal of trying to understand a healthy and disease state in incredible detail. It involves sequencing someone's genome, trying to get a complete picture of their molecular profile, by determining their DNA methylome, transcriptome, proteome, metabolome, auto-antibodyome, and their microbiome in many different locations in the body.

The ultimate goal is to have a very detailed assessment of a person's physiological state so that we can catch that state when it goes aberrant; that is, when someone has a predisposition for a disease or is on the verge of developing it.

\section{Have there been any recent advances in this field?}

As for new advances, there's been a huge number. For example, the ability to sequence genomes at an affordable cost, and the ability to measure complete transcriptomes using RNA sequencing. Proteomics has had huge advances, as has metabolomics, thanks to advances in mass spectrometry. One can get a much more complete and accurate picture of all these different 'omes' than we could even a few years ago. Also the price has dropped quite a bit. It's not super cheap, but it's in a range that you can now do this as a research project. The whole thing has only become possible in the past few years.

\section{Has your own attitude towards omics profiling changed} since the study was published?

I would say the answer is no. I've always been enthusiastic about trying to launch this project. It really only became possible because of the drop in cost of the various technologies and the ability to collect all the expertise in one location. The first version of omics profiling involved following about 40,000 molecules in my blood; the latest follows billions of individual measurements. This project is still evolving, but our ability to see things at this level has never been possible before, although I always envisioned it would be some day.

\section{What advice would you offer to people who are interested in having their own profile studied?}

At the moment it really is a research project. It's not routinely performed in the clinic because it involves taking lots of measurements, a complex analysis, and time to understand the data. My own view is that in the long run there will be some clinical form of this that involves hundreds or thousands of measurements. Right now, when you go to the doctor's office, you get about 15 things measured in a routine blood test. So, what we're trying to do is add tens of thousands, if not millions or billions of measurements. When it is applied in the clinic, it is probable that a smaller number of very useful markers would be used. It is just that we don't know what those markers are yet. 


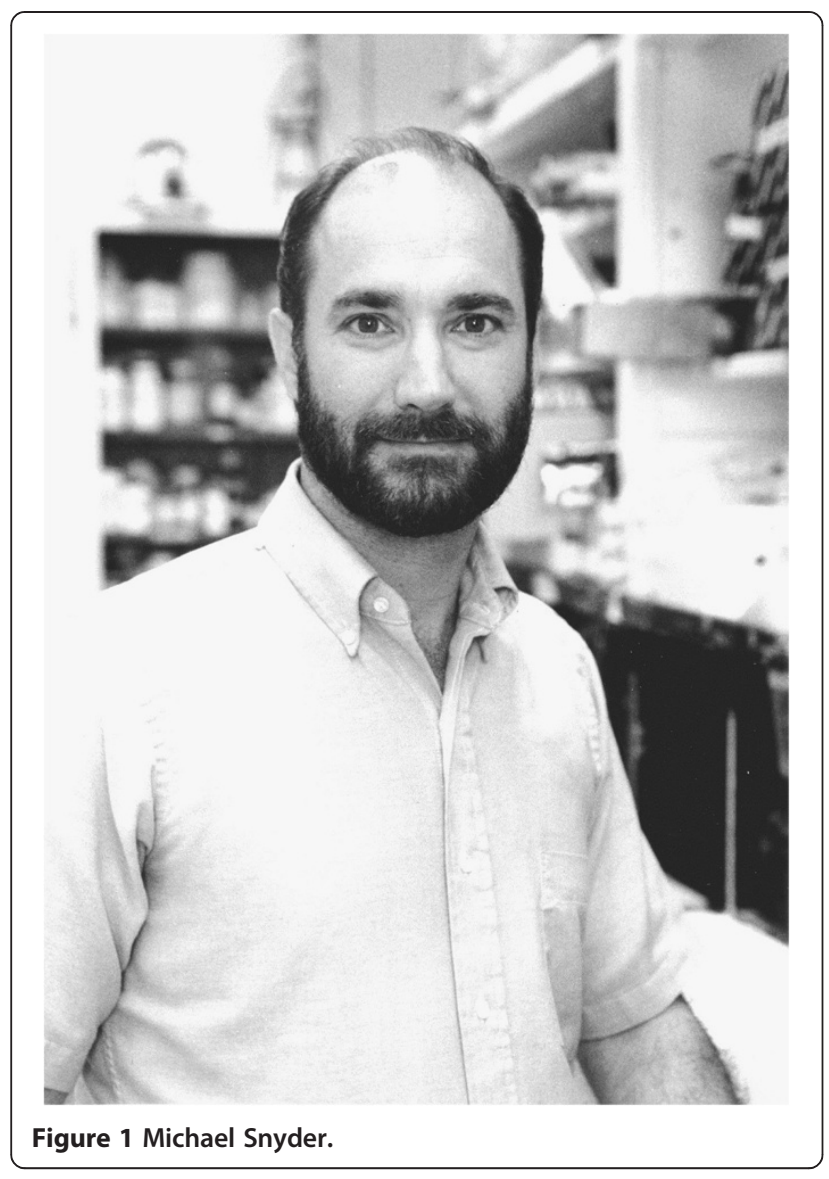

Do you think that omics profiling will be routinely used in the clinic in future?

Not in the form we are doing it. At the moment we have a very incomplete picture of what's going on, whereas if we were able to make thousands of measurements we would have a much better feeling. We just don't know, for the clinical tests, which thousand measurements are going to be most useful. We'll need certain measurements for diabetes, others for cancer, and specific tests will probably reveal themselves useful for different diseases.

I would like to see this ultimately used as a home test where an individual would prick their finger and make literally thousands of measurements to assess their health at high frequency, maybe once a month. We really need more frequent and comprehensive tests to follow someone's health, rather than the current practice of having individuals go to the doctor every two to three years to get a standard medical test, which to my mind is just woefully inadequate.

As we move toward healthcare that incorporates omics profiling, what do you think will be the main challenges? By the time it becomes a clinical test, the patient will simply be the recipient of it. For the moment, the biggest challenge is cost and who pays. Right now, because it's a research project, nobody is going to reimburse or cover it. Ultimately, when it becomes clear that making more detailed measurements saves money by actually preventing disease, then I think this will be better received, especially if we can set up low-cost tests. I think some degree of omics profiling could occur for a few hundred dollars in the future. I do think there will be some routine version that will be sufficiently cheap that people would just pay for it out of their own pockets, much like many pay for a prescription for a statin.

How do you think the relationship between patients, researchers, clinicians, and the general public might need to change?

This relationship will change quite a bit, and it is changing already. People don't realize that doctors really have about 15 minutes to look at your profile, and make recommendations about the next steps for your health. I don't see that (the time) changing a whole lot; physicians really don't have the time. I think you're going to see the burden shift to the patient because nobody else has the time to spend on your health like you do.

I think doctors are going to have to work with genomicists, who will be interpreting DNA. They'll have to work with specialists who make recommendations about whether you should be seeing a cardiologist or a nutritionist for your metabolic conditions, based on your DNA or omics profile. The patient really has to become more involved, and the role of the physician will be to help coordinate that process by making sure that patients are seeing the right people, and getting the right advice.

\section{How will the relationship between researchers and the general public change?}

I think we, as researchers, need to educate the public better. The public are very eager to learn. Everyone knows something about their DNA or genes. I think we have to learn not to be scared of using information to help ourselves; a lot of people are scared about genetic information and what it might mean. We should be trying to understand genomic information to manage healthcare better.

We might have to be careful in terms of the information that is relayed back. But I really do think that it is up to the individual to decide. Individuals are the gateway to their own genomic information; they should have access to the genomic information that they feel comfortable with handling. Obviously, they do have to receive proper genetic counseling.

As a researcher and a research participant what are your thoughts on participatory medicine?

Oh absolutely this is a good thing. We are all subjects in an ongoing research trial. What is going to happen is 
we're going to see the world of big data and data-driven medicine. As we collect information on individuals, whether it's clinical or research data, this will be shared in a common database. As that database grows large enough to have information about millions of people, we should be able to make data-driven, rather than intuitive, health decisions.

To explore this concept further, imagine a world where there are a million genomes and electronic health records. There's no question we will soon be in a world where we will be able to make links between treatments and genetic information. I would like to see a million longitudinal omic profiles like the one done on me where we're looking at a wide range of measurements. Then, we would have a huge number of biomarkers to better assess individuals' health states.

We would be so much more informed compared with now. I would argue that we're in the Stone Age compared with where we're going to be 20 years from now.

What significant advantages and challenges do you think remain for the implementation of a participatory model of healthcare?

I'd say the biggest challenge is a lack of research funding for pilot studies, such as the one that we have launched [1]. We are also going to need a good reimbursement model, by which patients are reimbursed for getting their exomes and their genomes sequenced. People should get a discount on their healthcare if they get their genome sequenced because, I think, they would be better placed to get diseases identified and diagnosed earlier. In principle, that is what should happen because people who get their exome or genome sequenced will be able to make health and lifestyle adjustments accordingly based on their disease risks.

\section{Competing interests}

Dr Snyder is founder and member of the scientific advisory board of Personalis, and a member of the scientific advisory board for Genapsys and AxioMx.

\section{Reference}

1. Chen R, Mias Gl, Li-Pook-Than J, Jiang L, Lam HY, Chen R, Miriami E, Karczewski KJ, Hariharan M, Dewey FE, Cheng Y, Clark MJ, Im H, Habegger L, Balasubramanian S, O'Huallachain M, Dudley JT, Hillenmeyer S, Haraksingh R, Sharon D, Euskirchen G, Lacroute P, Bettinger K, Boyle AP, Kasowski M, Grubert F, Seki S, Garcia M, Whirl-Carrillo M, Gallardo M, et al: Personal omics profiling reveals dynamic molecular and medical phenotypes. Cell 2012, 148:1293-1307. 